INVESTIGACIÓN

Recibido: 01/07/2021--- Aceptado: 16/09/2021--- Publicado: 03/01/2022

\title{
APLICACIÓN DE LAS TÉCNICAS DE MARKETING SENSORIAL EN LOS ESTABLECIMIENTOS DE MODA: EL CASO DE ZARA Y STRADIVARIUS
}

\author{
Application of sensory marketing techniques in fashion shops: the case of Zara \\ and Stradivarius
}

Pedro Pablo Marín Dueñas: Universidad de Cádiz. España. pablo.marin@uca.es

Diego Gómez Carmona: Universidad de Cádiz. España. diego.gomezcarmona@uca.es

\section{Cómo citar el artículo:}

Marín Dueñas, P. P. y Gómez Carmona, D. (2022). Aplicación de las técnicas de marketing sensorial en los establecimientos de moda: el caso de Zara y Stradivarius. Vivat Academia. Revista de Comunicación, 155, 17-32. $\underline{\text { http://doi.org/10.15178/va.2022.155.e1392 }}$

\section{RESUMEN}

La creciente importancia de los portales de compra online frente al retail físico, hace necesario el desarrollo de estrategias en el punto de venta para que los consumidores sigan acudiendo a las mismas a comprar. $\mathrm{Y}$, en este sentido, una de las grandes diferencias (por no decir el punto fuerte) entre la tienda física y la online es la experiencia física de poder tocar y sentir el producto. La tienda no debe ser entendida solo como un punto de venta, sino que se debe configurar como un espacio donde vivir experiencias. Y es aquí, integrada dentro de la estrategia de trade marketing, donde cobra especial relevancia el marketing de los sentidos. Partiendo de esta premisa, la presente investigación se centra en el estudio del marketing sensorial utilizado por las tiendas de moda del grupo Inditex Stradivarius y ZARA y, más concretamente, en como aplican estas técnicas en sus tiendas, desde el punto de vista del olfato, el oído, la vista y el tacto. Para ello se aplicará la metodología del análisis de contenido. De los resultados se desprende que estos establecimientos aplican de manera activa las técnicas de marketing sensorial, estando presentes en

\footnotetext{
1 Pedro Pablo Marín Dueñas: Doctor por la Universidad de Cádiz. Profesor Ayudante Doctor en el Departamento de Marketing y Comunicación de la Universidad de Cádiz. Coordinador de Grado en Marketing e Investigación de Mercados de la Universidad de Cádiz
} 
todas las tiendas, al objeto de mejorar la experiencia de compra de los consumidores.

PALABRAS CLAVE: Marketing sensorial - Neuromarketing - Retail Marketing Trade marketing - Merchandising - Moda - Punto de venta - Sentidos - Inditex

\section{ABSTRACT}

The growing importance of online shopping portals compared to physical retail makes it necessary to develop strategies at the point of sale to continue to go there to buy. In this sense, one of the big differences (if not the strongest point) between the physical shop and the online store is the physical experience of being able to touch and feel the product. The shop should not only be understood as a point of sale but should also be configured as a space in which to live experiences. Moreover, it is integrated within the trade marketing strategy, where the senses' marketing takes on particular relevance. Based on this premise, this research focuses on the study of sensory marketing used by the fashion shops of the Inditex group Stradivarius and ZARA and, more specifically, on how they apply these techniques in their shops, from the point of view of smell, hearing, sight and touch. In order to carry out the analyses, the content analysis methodology will be applied. The results show that these establishments actively apply sensory marketing techniques, being present in all the shops to improve the shopping experience for consumers.

KEYWORDS: Sensory marketing - Neuromarketing - Retail marketing - Trade marketing - Merchandising - Fashion - Point of sale - Senses - Inditex

\section{APLICAÇÃO DAS TÉCNICAS DE MARKETING SENSORIAL NAS LOJAS DE MODA: O CASO ZARA E STRADIVARIUS.}

\section{RESUMO}

A crescente importância dos portais de compra online em comparação com o retail físico, faz necessário o desenvolvimento de estratégias no ponto de venda para que os consumidores continuem frequentando as lojas para comprar. E, nesse sentido, uma das grandes diferenças(para não dizer o ponto forte) entre a loja física e a online é a experiência física de poder tocar e sentir o produto. A loja não deve ser entendida somente como um ponto de venda, mas deve ser entendido como um espaço onde viver experiências. E, é aqui onde é incluída dentro da estratégia de trade marketing, e cobra especial relevância o marketing das sensações. Partindo desta ideia, a presente pesquisa se centra no estudo do marketing sensorial utilizado pelas lojas de moda do grupo Inditex Stradivarius e ZARA, e mais concretamente, em como aplicam as técnicas nas suas lojas, desde o ponto de vista do cheiro, ouvido, vista e tato.Para isto se aplicará a metodologia de análise de conteúdo. Para isto se aplicará a metodologia de análise de conteúdo. Dos resultados se desprende que estas lojas aplicam de forma ativa as técnicas de marketing sensorial, estando presente em todas 
Marín Dueñas, P. P. y Gómez Carmona, D.

Aplicación de las técnicas de marketing sensorial en los establecimientos de moda: el caso de Zara y Stradivarius

as lojas, com o propósito de melhorar a experiência de compra dos consumidores.

\section{PALAVRAS CLAVE}

Marketing sensorial - Neuromarketing - Retail Marketing - Trade marketing Merchandising - Moda - Ponto de venda - Sentidos - Inditex

\section{INTRODUCCIÓN}

El retail de moda se enfrenta en la actualidad a una serie de problemas derivados, principalmente, del desarrollo de las nuevas tecnologías. Por un lado, el auge de la venta online que permite al comprador acceder a los productos a través de las pantallas de sus ordenadores, móviles o tablets y, por otro, el ilimitado acceso a la información que Internet permite y que convierte al consumidor en un usuario mucho más informado y exigente que ya no se limita a la compra de productos, sino que busca disfrutar de la experiencia de compra. Además, la globalización lleva aparejada la sobresaturación de los mercados en los que se pueden encontrar productos de difícil diferenciación y muy competitivos. Ante esta difícil situación la tienda física tiene que dar una respuesta efectiva para que el consumidor visite el punto de venta y compre en él. En definitiva, se trata que el sector retail evolucione adaptando su gestión de merchandising a las nuevas necesidades de los consumidores, creando experiencias de compra que conecten con el cliente en un plano emocional.

Y en esta evolución de la gestión del punto de venta ha surgido el denominado marketing sensorial como herramienta vinculada al marketing experiencial que busca crear experiencias de compra a través de las que un consumidor pueda vivir un momento único durante el tiempo que esté en el establecimiento generando en el mismo reacciones de tipo emocional que mejoren las relaciones marca-consumidor. De hecho, para autores como Segura y Sabaté (2008) la elección final del consumidor estará determinada por las emociones que en él despierte el proceso de compra. Para despertar dichas emociones el marketing sensorial busca la diferenciación a través de la vista, el oído, el gusto, el tacto y el olfato (de Garcillán López, 2015)

De hecho, el desarrollo cada vez mayor de estas acciones en los establecimientos comerciales se ve influido por las investigaciones que han demostrado que los humanos recuerdan más los impactos sensoriales vinculados a las emociones, haciendo estos más perdurables. Y esto es algo que no puede ofrecer el comercio online, por lo que las tiendas de moda deben aprovechar esta ventaja ofreciendo experimentar esa sensación en sus locales reforzando las emociones de los compradores.

En definitiva, los consumidores no sólo se ven influenciados en sus decisiones de compra por el precio o la calidad del producto, sino que variables como la ambientación del local, el olor, la percepción de los colores asociados al

Vivat Academia. Revista de Comunicación. 2022, nº 155, 17-32 
establecimiento o simplemente el llevarse una buena impresión en cuanto a la limpieza y el orden del local pasan a tener una importancia decisiva en el acto de compra, que ya no se considera como algo únicamente racional (de Garcillán López, 2015).

Todo esto ha derivado en un aumento de la actividad académica en esta área de conocimiento en los últimos tiempos (Schmitt, 2007; Hultén, 2011; Gómez y García, 2014) y este trabajo pretende seguir profundizando en esta línea de investigación a partir del análisis de la gestión que de estas técnicas que involucran a los sentidos hacen los establecimientos de moda.

\subsection{Definiendo el marketing de los sentidos}

De Garcillán López (2015) establece una diferenciación entre el marketing tradicional y el marketing de los sentidos partiendo de la racionalidad del primero frente a la importancia que las experiencias y las emociones tienen para los consumidores, que se comportan más guiados por sus impulsos que por sus razones. Frente a la idea de que el comportamiento del consumidor se basa en la satisfacción de las necesidades a partir de una oferta adecuada el marketing sensorial coloca a la emoción como eje sobre el que gira el mismo y centra el proceso de compra en la experimentación de sensaciones vinculadas a dicho proceso.

Así, el marketing de los sentidos permite influir en la percepción, juicios y comportamientos de los consumidores creando un ambiente agradable que incremente el tiempo de compra en el punto de venta (Berčík Et al., 2020; Jiménez, Bellido y López, 2019; Bilek, Vietoris e Ilko, 2016; Tauferova Et al., 2015). Esta técnica, supone una ventaja de los detallistas frente al comercio online. Los consumidores pueden experimentar a través de sus sentidos la experiencia en tiendas físicas, especialmente en aquellos productos que requieren tocar, sentir y probar previamente a la decisión de compra (Kim Et al., 2020). Específicamente, aquellos minoristas que se dedican al retail de moda tienen otra ventaja dado que a diferencia de otros bienes que no requieren evaluación física antes de su adquisición, la ropa debe verse, tocarse y generar una experiencia previa antes de decidir su compra (Kim Et al., 2020). Esta carencia de experiencia en el entorno online, provoca una tasa de devoluciones superior al 40\% (CNBC, 2016).

Considerando este dato, los establecimientos de moda deberían crear experiencias sensoriales en las que se pusiesen en práctica estrategias y tácticas que involucrasen a los cinco sentidos ya que su implementación de manera conjunta influye de manera decisiva en el comportamiento de compra (Hulten, Broweus y Van Dijk, 2008).

- Marketing aromático

Entre las herramientas de marketing sensorial encontramos el marketing aromático, un factor de gran influencia en el comportamiento de compra de los consumidores. Una investigación actual afirma que el $75 \%$ de nuestras emociones

Vivat Academia. Revista de Comunicación. 2022, nº 155, 17-32 
diarias están relacionadas con los aromas (Berčík Et al., 2020). Estas emociones influyen en el recuerdo generado por el estímulo y permite recordar algo que olemos mejor que algo que vemos, oímos o tocamos (Vlahos, 2007). En este sentido, las empresas pueden facilitar el reconocimiento del producto vinculando su identidad corporativa con un aroma y así diferenciar la marca de sus competidores.

De hecho, Conick (2017) afirma que percibir un olor agradable mejora un $44 \%$ el tiempo que un consumidor pasa en un establecimiento comercial a lo que Spectrio (2020) añade que influye, también, en el incremento del ticket de compra.

Un uso adecuado del marketing aromático ayuda a la mejora de las percepciones que los consumidores tienen de la marca, del punto de venta y de los propios productos, logrando, en definitiva, que las visitas a los establecimientos de moda se incrementen (ScentAir, 2020).

- Marketing háptico

Otra de las herramientas de marketing sensorial que pueden emplear los detallistas de ropa en sus establecimientos es el marketing háptico, un aspecto fundamental para la industria de la moda pues un consumidor que no puede tocar la prenda ni sentirla difícilmente la va a comprar. De hecho, es ésta una de las principales ventajas que los establecimientos de moda tienen en relación a la compra de moda online. El consumidor prefiere realizar sus compras en tiendas físicas porque pueden tocar y percibir la ropa haciéndose así una idea real de su calidad, su suavidad o dureza, de su peso o de las texturas con las que están producidas (TimeTrade, 2017; Klatzky, Lederman y Matula, 1993; Klatzky y Lederman, 1992).

En este sentido, es la suavidad la característica que más se valora a la hora de definir la calidad de un producto por parte de los consumidores de moda. Y para ser capaces de evaluarla las personas tienen que poder testarla en su propio cuerpo probándose la ropa, cosa que únicamente pueden hacer en una tienda (Monitor TM 2017; Chang Et al., 2015; Spence Et al., 2014; Workman, 2010; Childers y Peck, 2010).

\section{- Marketing musical}

Los especialistas en marketing utilizan la música para trasladar mensajes comunicacionales a los visitantes a las tiendas pasando a ser ésta un elemento más de la atmósfera comercial (Reynolds-Mcllnay y Morrin, 2019; Biswas, Lund y Szocs 2019; Roschk, Loureiro y Breitsohl, 2017) aumentando, además, la confianza que el consumidor tiene en el establecimiento (Guenzi, Johnson y Castaldo, 2009).

Así, los principales elementos musicales como la armonía o el timbre, la frecuencia o tono y la amplitud del sonido, percibida a través del volumen (Bruner, 1990; Lowe Et al., 2018; Krishna, 2013) son tenidos en cuenta a la hora de seleccionar un tipo de música $\mathrm{u}$ otro para que suene en el local. De hecho, esta decisión es muy importante y su elección suele ser de lo más variada. Por ejemplo, puede utilizarse la misma música en todo el establecimiento mientras que son muchos los puntos de venta que 
optan por zonificar la música que reproducen a lo largo de la superficie comercial teniendo en cuenta el tipo de producto que se comercializa en cada una de ellas (Cleaver, 2016; Haugh, 2016).

En cuanto al efecto que el uso de la música tiene sobre el comportamiento del consumidor los estudios ofrecen diferentes resultados (Baumann y Gayer, 2017). Mientras hay investigaciones que confirman que la utilización de música incrementa 15 minutos la presencia del cliente en el local (Andersson Et al., 2012; Sullivan, 2002) se pueden encontrar estudios que no han hallado diferencias significativas en el comportamiento entre utilizar música o no hacerlo (Wilson, 2003; North Et al., 2003).

Por otro lado, hay autores que sostienen que la reproducción de música en el punto de venta mejora el estado de ánimo de los consumidores haciendo que éstos se sientan mejor y provocando que consuman más y gasten más dinero (Baumann y Gayer, 2017), si bien estos resultados no han sido son confirmados por otros trabajos (North Et al., 2016; Milliman, 1982).

En definitiva, la investigación académica en este campo se ha centrado en analizar la selección musical, la implementación de la misma en el establecimiento y, por supuesto, en la influencia de la música en el comportamiento de los consumidores (Baumann y Gayer, 2017).

- Marketing visual

La modalidad visual suele ser la herramienta sensorial más empleada siendo, a menudo el de la vista el más importante de todos los sentidos (Biswas Et al., 2019; Biswas Et al., 2014).

Desde la perspectiva del análisis del comportamiento el modelo estímuloorganismo-respuesta (Koo y Kim, 2013) es utilizado frecuentemente para medir como influyen los estímulos visuales del establecimiento en los consumidores y, más concretamente, qué impacto tienen en su percepción, emoción y comportamiento de compra (Morone Et al., 2018; Kühn y Petzer, 2018).

Por otro lado, el color (Bagchi y Cheema 2013; Mehta y Zhu, 2009), la orientación de la cartelería (Romero y Biswas, 2016), la disposición del mobiliario (Jiang Et al., 2016) o el dinamismo en la ubicación y rotación de productos (Roggeveen Et al., 2015; Krishna Et al., 2017) son también factores habitualmente estudiados en las investigaciones sobre marketing visual.

Rodríguez (20018) afirma que aquellos establecimientos de moda que implementan estrategias de marketing visual mejoran el reconocimiento de marca, mejorando el posicionamiento e influyendo de manera positiva en la decisión de compra. Jang, Baek, Yoon \& Choo (2018) analizaron, por su parte, el concepto de complejidad visual y sus efectos sobre el placer a la hora de realizar una compra encontrando que cuando el grado de implicación del comprador es bajo, el efecto es 
negativo si bien, esta complejidad visual se relaciona con un incremento de la excitación emocional.

En definitiva, para conseguir un reconocimiento entre los consumidores que potencie el posicionamiento de la marca y, por tanto, aumente el nivel de las compras en los establecimientos del sector moda es importante despertar las emociones en el punto de venta a través del desarrollo de estrategias de marketing visual (Rodríguez, 2018).

\section{OBJETIVOS}

El objeto de estudio de la presente investigación es el sector retail de moda y, más concretamente, la aplicación de las técnicas de marketing sensorial por parte de los establecimientos de moda del grupo Inditex.

Derivado de dicho objeto de estudio, el objetivo general del estudio es analizar las acciones de marketing sensorial que las tiendas de moda implementan en el punto de venta para influenciar el comportamiento del consumidor. Específicamente se analizará el marketing visual, el marketing olfativo, el marketing auditivo, el marketing táctil o háptico en las tiendas ZARA y Stradivarius.

\section{METODOLOGÍA}

La herramienta metodológica que se ha usado para dar respuesta a los objetivos que se han marcado es la observación, definida por Posada (2001) como

La forma más sistematizada y lógica para el registro visual y verificable de lo que se pretende conocer; es decir, es captar de la manera más objetiva posible, lo que ocurre en el mundo real, ya sea para describirlo, analizarlo o explicarlo desde una perspectiva científica (p.5)

Para su aplicación, se ha elaborado una ficha de análisis a partir de las variables que se definen a continuación (tabla 1) y que se fundamentan en los trabajos de Manzano Et al. (2012), Morgan (2016), Palomares (2009) y Díez de Castro Et al. (2006). $\mathrm{Su}$ aplicación en los puntos de venta objeto del estudio (las tiendas ZARA y Stradivarius situadas en el Centro Comercial Área Sur, en Jerez de la Frontera) permitirá estudiar en primera persona cuáles son los estímulos del marketing sensorial que están presentes en los mismos y cómo se ha configurado la atmósfera del establecimiento para convertir el proceso de compra en una experiencia memorable. Se han seleccionado estas dos enseñas del grupo Inditex por dirigirse a dos tipos de consumidores con características bien diferenciadas. 
Marín Dueñas, P. P. y Gómez Carmona, D.

Aplicación de las técnicas de marketing sensorial en los establecimientos de moda: el caso de Zara y Stradivarius

Tabla 1: Variables de análisis

\begin{tabular}{|c|c|c|}
\hline \multirow[t]{3}{*}{$\begin{array}{l}\text { MARKETING } \\
\text { VISUAL }\end{array}$} & $\begin{array}{l}\text { Color en el } \\
\text { establecimiento }\end{array}$ & $\begin{array}{l}\text { Que se medirá a través del tono predominante en el } \\
\text { mismo y que puede ser oscuro/claro, } \\
\text { luminoso/apagado, o de colores }\end{array}$ \\
\hline & $\begin{array}{l}\text { Iluminación en } \\
\text { el } \\
\text { establecimiento }\end{array}$ & $\begin{array}{l}\text { Medida en diferentes zonas del espacio: entrada, zona } \\
\text { de pago, probadores, escaparate, expositores y } \\
\text { estanterías, resto de la superficie comercial y que } \\
\text { pueden estar iluminadas con una luz cálida, fría o } \\
\text { luces de colores, y que pueden estar enfocadas o } \\
\text { degradadas }\end{array}$ \\
\hline & $\begin{array}{l}\text { Disposición del } \\
\text { establecimiento }\end{array}$ & $\begin{array}{l}\text { Definida a través del tipo de distribución, los } \\
\text { elementos que configuran el espacio y la atmósfera } \\
\text { comercial. Se medirá en el recorrido de la planta, en los } \\
\text { expositores y estanterías, en el mobiliario y en el } \\
\text { escaparate }\end{array}$ \\
\hline $\begin{array}{l}\text { MARKETING } \\
\text { OLFATIVO }\end{array}$ & Olor & $\begin{array}{l}\text { Medido a través de la intensidad (fuerte o suave) y el } \\
\text { tipo de aroma (floral, frutal, dulce, cítrico...) }\end{array}$ \\
\hline $\begin{array}{l}\text { MARKETING } \\
\text { AUDITIVO }\end{array}$ & Música & Estilo, tempo, ritmo y volumen \\
\hline \multirow[t]{4}{*}{$\begin{array}{l}\text { MARKETING } \\
\text { TACTIL }\end{array}$} & $\begin{array}{l}\text { Orden en el } \\
\text { establecimiento }\end{array}$ & \multirow[t]{4}{*}{ Adecuado/no adecuado } \\
\hline & $\begin{array}{l}\text { Disposición de } \\
\text { las prendas }\end{array}$ & \\
\hline & Trato personal & \\
\hline & $\begin{array}{l}\text { Limpieza en el } \\
\text { establecimiento }\end{array}$ & \\
\hline
\end{tabular}

Fuente: Elaboración propia a partir de Manzano Et al. (2012), Morgan (2016), Palomares (2009) y Díez de Castro Et al. (2006)

\section{RESULTADOS}

Desde el punto de vista del marketing visual la fachada de ZARA destaca por sus grandes dimensiones, su iluminación a través de luz blanca y un estilo arquitectónico moderno y atractivo. El escaparate está formado por cinco cristaleras de grandes dimensiones dirigidas a atraer a los distintos grupos de consumidores (2 a hombres, 2 a mujeres y 1 a niños) y decorados con maniquíes vestidos con la ropa de la temporada vigente. La entrada, por su parte, destaca por sus tres grandes puertas metálicas que invitan a entrar al paseante al estar abiertas de par en par. Una vez en el interior de la tienda, se puede ver un mobiliario y una decoración moderna y elegante formada por mesas, espejos y estanterías de metal y de color negro iluminadas con luz blanca si bien, en la zona central del establecimiento, se observan algunas mesas y percheros que dan cierta sensación de desorden. Igualmente, el local es muy luminoso aunque la iluminación del interior puede resultar molesta a causa de la gran cantidad de focos de luz blanca distribuidos por todo el establecimiento. En este sentido, en todo el local destaca la luz fría salvo en la zona de pago, el único punto del local en el que se puede encontrar una luz amarilla cálida.

Vivat Academia. Revista de Comunicación. 2022, nº 155, 17-32 
En el caso de Stradivarius, también está formado por un escaparate en el que destacan una gran cristalera (si bien en este caso está sólo dedicado a ropa femenina, dando sensación de sencillez y orden y permitiendo ver bien las prendas expuestas) y una gran entrada, dividida en dos por una columna y coronadas por el logotipo iluminado del negocio. Respecto al mobiliario, es moderno pero sencillo y está compuesto de sistemas murales, estanterías, mesas de madera, percheros circulares de pie y percheros lineales colgados en las paredes, así como espejos. El problema es la disposición del mismo que no sigue un patrón claro como en el caso de ZARA y provoca que el movimiento por el establecimiento no sea fluido. En cuanto a la iluminación, también predomina la luz blanca con focos que iluminan de manera directa y exclusiva tanto a los expositores como a las estanterías. Lo mismo ocurre con la iluminación del escaparate en la que los focos se centran en los maniquíes expuestos. Por el contrario, la zona de probadores no está muy iluminada y da sensación de agobio y desorden.

En lo referente al marketing olfativo, en el caso de ZARA el perfume que se utiliza en la tienda es uno de los que ellos comercializan y que en ese momento está de promoción (concretamente el producto Wonder Rose). En este caso se trataba de un olor dulce con cierto toque floral. La fragancia era pulverizada por el propio personal cada cierto tiempo y en las distintas zonas del establecimiento. Stradivarius, por su parte, usa un aroma que es muy característico y que está desarrollado exclusivamente para las tiendas de la firma por lo que es un olor único (odotipo). Se trata de un olor dulce pero muy intenso y que se percibe incluso a metros de la entrada. En este caso, la fragancia se vaporiza de manera mecánica por todo el establecimiento cada media hora.

A lo largo de todo el establecimiento ZARA suena la misma música de estilo pop británico y no se diferencia por zonas. El ritmo de las canciones era rápido incitando al consumidor a moverse por la planta de manera acelerada, si bien el volumen al que sonaba no era muy alto. En Stradivarius, además de pop también sonaron temas del género techno y house. En todos los casos tanto el tempo como el ritmo de la música era rápido y el volumen más alto que en ZARA, si bien no llegaba a ser molesto. En cualquier caso, la música reproducida se adecuaba al target de consumidor de las tiendas.

Finalmente, en cuanto al marketing táctil en ZARA la textura y suavidad de las prendas así como la disposición de las mismas incita a tocarlas. Además, el personal del establecimiento está constantemente doblando y ordenando la ropa por lo que la sensación de limpieza y organización es muy positiva, incluida la zona de probadores. Igualmente, el trato personal es cordial y la atención a los clientes tanto a la hora de que asesoren como a la hora de solicitar ayuda en busca de alguna prenda o alguna talla concreta es muy bueno.

En Stradivarius la situación no es tan óptima. Si bien en general la limpieza y el orden son buenos sí que se nota cierto grado de descuido, con ropa desordenada que

Vivat Academia. Revista de Comunicación. 2022, nº 155, 17-32 
no ha sido doblada ni colocada en su lugar en bastante tiempo, sensación que se acrecienta en los probadores en los que se nota incluso una sensación de desinterés por organizar las prendas que los clientes van dejando y que incluso se acumula en los propios probadores. En cuanto al trato personal, en este caso tampoco los dependientes están atentos a los usuarios ni les asesoran de manera directa siendo el usuario el que tiene que dar vueltas por el establecimiento para encontrar a un dependiente y solicitarle la ayuda, cosa que no ocurre en ZARA, si bien el trato es correcto y cordial.

\section{DISCUSIÓN}

Los resultados alcanzados en nuestra investigación fruto de la observación demuestran que el desarrollo de las técnicas de marketing visual de ZARA como de Stradivarius aprovechan los recursos que tienen a su disposición, cuidando la fachada, los escaparates, la iluminación, los rótulos y los puntos de acceso. Es decir, la mayoría de aspectos relativos al marketing de entrada, orientado principalmente a un consumidor shopper que busca argumentos para visitar un establecimiento y no otro, están implementados. La ventaja de desarrollar estas técnicas es que facilita el acceso a este segmento de consumidores que por lo general se guían por el precio. Aplicando marketing de entrada es posible conseguir captarlo y fidelizarlo.

En el caso del marketing visual desarrollado en el interior del establecimiento, la investigación muestra que en ZARA y Stradivarius parece existir cierta sensación de desorden en la disposición del mobiliario. La literatura previa sobre libre disposición sugiere distribuir el mobiliario de forma aleatoria permitiendo visualizar distintos sectores de la tienda y una mayor cantidad de productos, posibilitando esta distribución un mayor tiempo de permanencia en el punto de venta y provocando un mayor volumen de compras de carácter impulsivo. Esto parece indicar que el desorden percibido puede estar causado a propósito. La iluminación más luminosa en la sección de exposición facilita la percepción de las prendas visualmente en la distancia mientras que la iluminación en la zona de pago, más atenuada, brinda al consumidor un momento de intimidad, creando un ambiente agradable a la hora de pagar.

En lo referente a los estímulos olfativos empleados por ambos establecimientos existen diferencias notables estando este aspecto menos cuidado en el caso de ZARA. La utilización no sistemática de un aroma puede indicar la búsqueda actual de esa fragancia que permita identificar la marca o que mejore el ticket medio de los consumidores. Sea como fuere parece que en el caso de ZARA la estrategia de marketing olfativo aún no está implementada completamente y que se centra más bien en promocionar sus perfumes que en conseguir una identidad olorosa. En contraste, Stradivarius utiliza claramente un odotipo vinculando un olor concreto a su marca que le otorga las ventajas típicas de estas estrategias. Es decir, le permite diferenciarse, mejorar el recuerdo generado por sus productos y la frecuencia de visita al punto de venta.

Vivat Academia. Revista de Comunicación. 2022, n 155, 17-32 
En lo referente a las estrategias auditivas, aunque los dos establecimientos emplean música en el interior sin zonificar, no aprovechan la comunicación directa (a través de megafonía, por ejemplo) en el punto de venta con el consumidor. De acuerdo con el cuerpo literario, utilizar este tipo de comunicación mejoraría la confianza del consumidor en el establecimiento y podría alentar las compras promocionales.

Por último, parece que la textura y suavidad presente en las prendas de ZARA mejora la percepción de calidad del establecimiento. Stradivarius, en cambio debería mejorar la colocación de las prendas facilitando a los consumidores su percepción háptica, la cual no es posible captar debido a la falta de organización. Considerando que parte de la calidad de la ropa es percibida por el tacto, dificultar al consumidor que los productos se puedan tocar implica una falta de información importante a la hora de formar juicios sobre la calidad de la ropa

\section{CONCLUSIONES}

Es un hecho que el desarrollo del marketing sensorial ha conseguido captar la atención de los investigadores del marketing generando un gran cuerpo literario durante los últimos años. La transferencia del conocimiento científico generado hacia el sector empresarial evidencia la aplicación cada vez más frecuente de técnicas sensoriales de marketing en los establecimientos comerciales. Concretamente los directivos de marketing utilizan distintas técnicas que permiten mejorar la experiencia del consumidor a través de sus sentidos. Es decir, la práctica del tradicional merchandaising visual está siendo canibalizada por el merchandaising de seducción, que implica a más de un sentido mejorando la sensación del consumidor en el punto de venta.

En el caso estudiado en este trabajo -si bien se debe seguir desarrollando el mismo ampliando la muestra de estudio- se puede concluir que hay margen para mejorar la implementación del marketing de los sentidos en los establecimientos de moda. Se debe seguir potenciando la aplicación de distintas acciones que estimulen los sentidos y que no solo atraigan al consumidor al establecimiento comercial, sino que consigan que las sensaciones, sentimientos y percepciones que éstas provoquen en ellos conviertan su proceso de compra en una experiencia atractiva y exclusiva. Solo de esta forma se estará en una mejor posición para ganar la partida a la venta online.

\section{REFERENCIAS}

Andersson, P. K., Kristensson, P., Wästlund, E., \& Gustafsson, A. (2012). Let the music play or not: The influence of background music on consumer behavior. Journal of retailing and consumer services, 19(6), 553-560. https://doi.org/10.1016/j.jretconser.2012.06.010

Vivat Academia. Revista de Comunicación. 2022, nº 155, 17-32 
Marín Dueñas, P. P. y Gómez Carmona, D.

Aplicación de las técnicas de marketing sensorial en los establecimientos de moda: el caso de Zara y Stradivarius

Bagchi, R., \& Cheema, A. (2013). The effect of red background color on willingnessto-pay: the moderating role of selling mechanism. Journal of Consumer Research, 39(5), 947-960. https://doi.org/10.1086/666466

Berčík, J., Mravcová, A., Gálová, J., \& Mikláš, M. (2020). The use of consumer neuroscience in aroma marketing of a service company. Potravinarstvo Slovak Journal of Food Sciences, 14, 1200-1210. https://doi.org/10.5219/1465

Bilek, M., Vietoris, V., \& Ilko, V. (2016). Shelf life extension and sensory evaluation of birch tree sap using chemical preservatives. Potravinarstvo Slovak Journal of Food Sciences, 10(1), 499-505. https://doi.org/10.5219/649

Biswas, D., \& Szocs, C. (2019). The Smell of Healthy Choices: Cross-Modal Sensory Compensation Effects of Ambient Scent on Food Purchases. Journal of Marketing Research, 56(1), 123-141. https://doi.org/10.1177/0022243718820585

Bruner, G. C. (1990). Music, mood, and marketing. Journal of marketing, 54(4), 94-104. Music, mood, and marketing.

Chang, H. J., Song, J., Yeo, C., \& Kim, J. (2015). Exploring factors influencing perceived quality on sportswear fabric. In International Textile and Apparel Association Annual Conference Proceedings, 72(1). University Digital Press.

Childers, T. L., \& Peck, J. (2010). Informational and affective influences of haptics on product evaluation: Is what I say how I feel? In A Krishna (ed.). Sensory Marketing: Research on the Sensuality of Products. Routledge/Taylor \& Francis Group, 63- 72.

Cian, L., Krishna, A., \& Elder, R. S. (2015). A sign of things to come: behavioral change through dynamic iconography. Journal of Consumer Research, 41(6), 14261446. https://doi.org/10.1086/680673

Conick, H. (2017). The past, present and future of AI in marketing. Marketing News, 51(1), 26-35.

CNBC (2016). A $\$ 260$ billion 'ticking time bomb': the costly business of retail returns. www.cnbc.com/2016/12/16/a-260-billion-ticking-time-bomb-the-costly-business -of-retailreturns.html

Cleaver, E. (2016). How to Help. Consumer Equality: Race and the American Marketplace: Race and the American Marketplace, 167.

De Garcillán López, M. (2015). Persuasión a través del marketing sensorial y experiencial. Opción, 31(2), 463 - 478. 
Marín Dueñas, P. P. y Gómez Carmona, D.

Aplicación de las técnicas de marketing sensorial en los establecimientos de moda: el caso de Zara y Stradivarius

Díez de Castro, E., Landa, F. y Navarro, A. (2006). Merchandising. Teoría y práctica. Ediciones Pirámide (Grupo Anaya, S.A).

Gómez, M. y García, C. (2014). The use of sensorial marketing in stores: attracting clients through the senses. En Musso, F, Y Druica, E. (eds.) (2014). Advances in Marketing, Customer Relationship Management, and E-Services.

Guenzi, P., Johnson, M. D., \& Castaldo, S. (2009). A comprehensive model of customer trust in two retail stores. Journal of Service Management, 20(3), 290-316. https://doi.org/10.1108/09564230910964408

Hultén, B. (2011). Sensory marketing: the multi-sensory brand-experience concept. European Business Review, 23(3), 256-273. https:// doi.org/10.1108/09555341111130245

Jang, J. Y., Baek, E., Yoon, S. Y., \& Choo, H. J. (2018). Store design: Visual complexity and consumer responses. International Journal of Design, 12(2), 105-118.

Jiang, Y., Gorn, G. J., Galli, M., \& Chattopadhyay, A. (2016). Does your company have the right logo? How and why circular-and angular-logo shapes influence brand attribute judgments. Journal of Consumer Research, 42(5), 709-726. https://doi.org/10.1093/jcr/ucv049

Jiménez, G., Bellido, E. y López, A. (2019). Marketing sensorial: el concepto, sus técnicas y su aplicación en el punto de venta. Vivat Academia. Revista de Comunicación, 148, 121-147. http://doi.org/10.15178/va.2019.148.121-147

Kim, J. H., Kim, M., Yoo, J., \& Park, M. (2020). Consumer decision-making in a retail store: the role of mental imagery and gender difference. International Journal of Retail \& Distribution Management. 49(3), 421-445. https://doi.org/10.1108/IJRDM10-2019-0353

Klatzky, R. L., \& Lederman, S. J. (1992). Stages of manual exploration in haptic object identification. Perception $\mathcal{E}$ Psychophysics, 52(6), 661-670.

Klatzky, R. L., Lederman, S. J., \& Matula, D. E. (1993). Haptic exploration in the presence of vision. Journal of Experimental Psychology: Human Perception and Performance, 19(4), 726.

Koo, W., \& Kim, Y. K. (2013). Impacts of store environmental cues on store love and loyalty: single-brand apparel retailers. Journal of International Consumer Marketing, 25(2), 94-106. https://doi.org/10.1080/08961530.2013.759044

Krishna, A. (2013). Introduction: What Is Sensory Marketing?. In Customer Sense, 1-18. Palgrave Macmillan.

Vivat Academia. Revista de Comunicación. 2022, nº 155, 17-32 
Marín Dueñas, P. P. y Gómez Carmona, D.

Aplicación de las técnicas de marketing sensorial en los establecimientos de moda: el caso de Zara y Stradivarius

Krishna, A., Lee, S. W., Li, X., \& Schwarz, N. (2017). Embodied cognition, sensory marketing, and the conceptualization of consumers' judgment and decision processes: introduction to the issue. Journal of the Association for Consumer Research, 2(4), 377-381. https:// doi.org/10.1086/694453

Kühn, S. W., \& Petzer, D. J. (2018). Fostering purchase intentions toward online retailer websites in an emerging market: An SOR perspective. Journal of Internet Commerce, 17(3), 255-282. https://doi.org/10.1080/15332861.2018.1463799

Lowe, J., Maggioni, I., \& Sands, S. (2018). Critical success factors of temporary retail activations: A multi-actor perspective. Journal of Retailing and Consumer Services, 40, 74-81. https:// doi.org/10.1016/j.jretconser.2017.09.005

North, A. C., Sheridan, L. P., \& Areni, C. S. (2016). Music congruity effects on product memory, perception, and choice. Journal of Retailing, 92(1), 83-95. https://doi.org/10.1016/j.jretai.2015.06.001

North, A. C., Shilcock, A., \& Hargreaves, D. J. (2003). The effect of musical style on restaurant customers' spending. Environment and behavior, 35(5), 712-718. https://doi.org/10.1177/0013916503254749

Manzano, R., Gavilán, D., Avello, M., Abril, C. y Serra, T. (2012). Marketing sensorial: Comunicar con los sentidos en el punto de venta. Pearson Educación.

Mehta, R., \& Zhu, R. J. (2009). Blue or red? Exploring the effect of color on cognitive task performances. Science, 323(5918),

https://doi.org/10.1126/science.1169144

Milliman, R. E. (1982). Using background music to affect the behavior of supermarket shoppers. Journal of marketing, 46(3), 86-91.

Monitor ${ }^{\mathrm{TM}}$. (2017). Getting It Right. https://lifestylemonitor.cottoninc.com/gettingit-right/

Morgan, T. (2016). Visual merchandising: escaparates e interiores comerciales (3a. ed.). Editorial Gustavo Gili.

Morone, A., Nemore, F., \& Schirone, D. A. (2018). Sales impact of servicescape's rational stimuli: A natural experiment. Journal of Retailing and Consumer Services, 45, 256-262. https:// doi.org/10.1016/j.jretconser.2018.09.011

Palomares, R. (2009). Merchandising: Teoría, práctica y estrategia. ESIC 
Marín Dueñas, P. P. y Gómez Carmona, D.

Aplicación de las técnicas de marketing sensorial en los establecimientos de moda: el caso de Zara y Stradivarius

Romero, M., \& Biswas, D. (2016). Healthy-left, unhealthy-right: Can displaying healthy items to the left (versus right) of unhealthy items nudge healthier choices? Journal of Consumer Research,43(1), 103-112. https://doi.org/10.1093/jcr/ucw008

Reynolds-McIlnay, R., \& Morrin, M. (2019). Increasing shopper trust in retailer technological interfaces via auditory confirmation. Journal of Retailing, 95(4), 128 142. https://doi.org/10.1016/j.jretai.2019.10.006

Rodríguez, C. L. (2018). The role of visual merchandising to position fashion retailers: a key place in Spanish Literature. aDResearch: Revista Internacional de Investigación en Comunicación, 17, 8-29. https://doi.org/10.7263/adresic-017-01

Roggeveen, A. L., Grewal, D., Townsend, C., \& Krishnan, R. (2015). The impact of dynamic presentation format on consumer preferences for hedonic products and services. Journal of Marketing, 79(6), 34-49. https://doi.org/10.1509/jm.13.0521

Roschk, H., Loureiro, S. M. C., \& Breitsohl, J. (2017). Calibrating 30 years of experimental research: a meta-analysis of the atmospheric effects of music, scent, and color. Journal of Retailing, 93(2), 228-240.

ScentAir. (2020). Engage Customers with Scent Marketing. https://scentair.com

Schmitt, B. H. (2007). Experiential marketing. Innovation, 3(5), 4.

Segura, C. y Sabaté, F. (2008). Marketing Experiencial: el marketing de los sentimientos y sus efectos sobre la mejora en la comunicación. XII Congreso de Ingeniería de Organización (págs. 267-272). II International Conference on Industrial Engineering and Industrial Management.

Spectrio (2020). Engage Your Customers. https://www.spectrio.com

Spence, C., Puccinelli, N. M., Grewal, D., \& Roggeveen, A. L. (2014). Store atmospherics: A multisensory perspective. Psychology \& Marketing, 31(7), 472-488.

Sullivan, M. (2002). The impact of pitch, volume and tempo on the atmospheric effects of music. International Journal of Retail \& Distribution Management, 30 (6), 323-330. https://doi.org/10.1108/09590550210429531

Tauferova, A., Tremlova, B., Bednar, J., Golian, J., Zidek, R., \& Vietoris, V. (2015). Determination of ketchup sensory texture acceptability and examination of determining factors as a basis for product optimization. International journal of food properties, 18(3), 660-669. https://doi.org/10.1080/10942912.2013.853186

Vivat Academia. Revista de Comunicación. 2022, nº 155, 17-32 
Marín Dueñas, P. P. y Gómez Carmona, D.

Aplicación de las técnicas de marketing sensorial en los establecimientos de moda: el caso de Zara y Stradivarius

TimeTrade (2017). The State of the Retail 2017. https://www.timetrade.com/resource/state-retail-report-2017

Vlahos, J. (2007) Scent and Sensibility. The New York Times. https://www.nytimes.com/2007/09/09/realestate/keymagaz ine/909SCENT$\underline{\text { txt.html }}$

Wilson, S. (2003). The effect of music on perceived atmosphere and purchase intentions in a restaurant. Psychology of music, 31(1), 93-112. https://doi.org/10.1177/0305735603031001327

Workman, L. (2010). Compulsive buying: a theoretical framework. The Journal of Business Inquiry, 9(1), 89-126.

\section{AUTOR/ES:}

\section{Pedro Pablo Marín Dueñas}

Doctor en Ciencias Sociales por la Universidad de Cádiz. Profesor Ayudante Doctor en el departamento de Marketing y Comunicación, en la Facultad de Ciencias Sociales y de la Comunicación de la Universidad de Cádiz, es coordinador del Grado en Marketing e Investigación de Mercados y del doble Grado en Marketing e Investigación de Mercados y Turismo de la Universidad de Cádiz. Es miembro investigador del Instituto de Investigación Universitario para el Desarrollo Social Sostenible y Director de la Radio Universitaria INDESS Media Radio. Su línea de investigación principal es la comunicación en las organizaciones, contando con más de 40 publicaciones tanto en revistas de impacto (JCR, SJR, ESCI) como en editoriales indexadas en el SPI.

Orcid ID: https:// orcid.org/0000-0001-8692-1174

\section{Diego Gómez-Carmona}

Doctor en Ciencias Económicas y Empresariales por la Universidad de Granada. Profesor Sustituto Interino en el departamento de Marketing y Comunicación, en la Facultad de Ciencias Sociales y de la Comunicación de la Universidad de Cádiz. Es miembro investigador del Instituto de Investigación Universitario para el Desarrollo Social Sostenible y forma parte de la Comisión de Investigación del INDESS. Su línea de investigación principal es la neurociencia del consumidor. Sus trabajos más recientes han aparecido en revistas indexadas en JCR, como Physiology \& Behavior o DYNA y se han presentado en conferencias internacionales como AEMARK o el Congreso Hispano-Lusitano de Gestión Científica.

Orcid ID: https://orcid.org/0000-0002-0146-5956

Nuestro agradecimiento al grupo de investigación SEJ-482 Innovación Social en Marketing de la Universidad de Cádiz 